\title{
Adaptive Constrained Control for Uncertain Nonlinear Time-Delay System with Application to Unmanned Helicopter
}

\author{
Rong Li $\mathbb{D},{ }^{1}$ Qingxian Wu $\mathbb{D},{ }^{1}$ Qingyun Yang $\left(\mathbb{D},{ }^{2}\right.$ and Hui Ye $\mathbb{D}^{3}$ \\ ${ }^{1}$ College of Automation Engineering, Nanjing University of Aeronautics and Astronautics, Nanjing 210016, China \\ ${ }^{2}$ School of Mechanical and Electrical Engineering, Zaozhuang University, Zaozhuang 277160, China \\ ${ }^{3}$ School of Electronics and Information, Jiangsu University of Science and Technology, Zhenjiang 212003, China
}

Correspondence should be addressed to Qingxian Wu; wuqingxian@nuaa.edu.cn

Received 30 June 2017; Revised 16 September 2017; Accepted 26 September 2017; Published 13 February 2018

Academic Editor: Libor Pekař

Copyright (c) 2018 Rong Li et al. This is an open access article distributed under the Creative Commons Attribution License, which permits unrestricted use, distribution, and reproduction in any medium, provided the original work is properly cited.

\begin{abstract}
This paper investigates a class of nonlinear time-delayed systems with output prescribed performance constraint. The neural network and DOB (disturbance observer) are designed to tackle the uncertainties and external disturbance, and prescribed performance function is constructed for the output prescribed performance constrained problem. Then the robust controller is designed by using adaptive backstepping method, and the stability analysis is considered by using Lyapunov-Krasovskii. Furthermore, the proposed method is employed into the unmanned helicopter system with time-delay aerodynamic uncertainty. Finally, the simulation results illustrate that the proposed robust prescribed performance control system achieved a good control performance.
\end{abstract}

\section{Introduction}

Time-delay systems have drawn considerable attention in the past decade [1,2]. The adaptive backstepping technology was employed into the uncertain nonlinear time-delay system in [3]. The dynamic surface method was presented for the nonlinear time-delay system in [4]. In [5], the nonlinear stochastic system with time delay was studied. The finitetime control method was proposed for a class of time-delay systems in [6, 7]. In the previous studies on time-delay system, the uncertain nonlinear systems consisting of both constraint and external disturbances were not considered. In this paper, we will study a class of uncertain nonlinear timedelay systems subject to constraint.

It is well known that the uncertainty and external disturbance have an effect on the tracking performance of closed systems. Neural network is popular for its ability to cope with uncertainty [8]. In [9], the neural network was introduced into a class of nonlinear systems with unknown coefficient matrices. Combining RBFNN (radial basis function neural network) and disturbance observer, fault tolerant control method was presented to deal with input saturated system with actuator faults in [10]. Moreover, the disturbance observer is a valid method to deal with external disturbance [11]. In [12], the disturbance observer was proposed for permanent-magnet synchronous motor drivers. In [13], the sliding mode disturbance observer was presented to deal with mismatched disturbance. In [14], the disturbance observer was employed into a transport aircraft control system subject to continuous heavy cargo airdrop. In this paper, the neural network and disturbance observer will be utilized to tackle uncertainties, time delay, and external disturbance.

Another challenging problem in controller design lies in the constrained condition of the nonlinear systems [15]. The existence of constraint condition may degrade the performance or cause the instability of the closed control systems [16]. Using the Barrier Lyapunov function and adaptive backstepping technology, a robust constrained controller for a class of nonlinear strict systems was presented in 
[17]. In [18], the Barrier Lyapunov function was employed into the switched systems subject to output constraints. In [19], the Barrier Lyapunov function and high-gain observer were introduced to deal with the constrained trajectory tracking problem of the marine surface vessel. Additionally, prescribed performance is another method to cope with output constraints, by defining the appropriate prescribed performance. In [20], the prescribed performance-based feedback linearization method was proposed to deal with output tracking error constraints for the MIMO (multipleinput multiple-output) nonlinear systems. In [21], the prescribed performance and adaptive fuzzy logic were employed into the nonlinear adaptive controller design. To the best of the authors' knowledge, there is still no research about uncertain nonlinear time-delay system considering uncertainties, external disturbance, and output constraints. Thus, in this paper, we will present a prescribed performancebased adaptive constrained control method for the time-delay nonlinear systems.

Nowadays, the unmanned helicopter system has received an increasing attention, and there is an amount of studies about the flight control approaches [22, 23]. In [24], chattering-free sliding mode was proposed for the miniature helicopter system. To solve the tracking problem with nonlinearity, the model predictive control method for unmanned helicopters was presented in [25]. In [26], a trajectory tracking control method was proposed for unmanned helicopter system with constraint conditions. However, with the increasing demands for real time and accuracy, the aerodynamic disturbance caused by transmission delay for unmanned helicopter control system cannot be ignored. In this paper, we will apply the prescribed performance-based robust adaptive control approach for the uncertain unmanned helicopter systems with external disturbance, time delay, and output constraints.

This paper is organized as follows. In Section 2, problem statement and preliminaries of time-delay system and prescribed performance are introduced. Section 3 presents the entire adaptive controller design and stability analysis. In Section 4, the prescribed performance-based control method is employed into the unmanned helicopter system. Finally, simulation and conclusion are given in Sections 5 and 6, respectively.

\section{Problem Statement and Preliminaries}

In this subsection, we will review some preliminary knowledge about nonlinear time-delay system, prescribed performance, and neural network, which are necessary in the following controller design. Firstly, consider a class of uncertain MIMO nonlinear time-delay systems in the form of

$$
\begin{aligned}
\dot{x}_{i}(t)= & f_{i}\left(\bar{x}_{i}(t)\right)+g_{i}\left(\bar{x}_{i}(t)\right) x_{i+1}(t)+h_{i}\left(\bar{x}_{i}\left(t-\tau_{i}\right)\right) \\
& +\Delta f_{i}\left(\bar{x}_{i}(t)\right)+d_{i}(t), \quad 1 \leq i \leq n-1 \\
\dot{x}_{n}(t)= & f_{n}\left(\bar{x}_{n}(t)\right)+g_{n}\left(\bar{x}_{n}(t)\right) u(t)+h_{n}\left(\bar{x}_{n}\left(t-\tau_{n}\right)\right)
\end{aligned}
$$

$$
\begin{aligned}
& \quad+\Delta f_{n}\left(\bar{x}_{n}(t)\right)+d_{n}(t) \\
& y(t)=x_{1}(t),
\end{aligned}
$$

where $x_{i}(t) \in R^{m}, i=1,2, \ldots, n$, are the system state vectors which are assumed to be measurable, $\bar{x}_{i}(t)=$ $\left[x_{1}^{T}(t), x_{2}^{T}(t), \ldots, x_{i}^{T}(t)\right]^{T} \in R^{m \cdot i}, y \in R^{m}$ is the output vector, and $u(t) \in R^{m}$ is the control input vector. $f_{i}\left(\bar{x}_{i}(t)\right) \in R^{m}, i=$ $1,2, \ldots, n$, are the known smooth nonlinear function, $g_{i}\left(\bar{x}_{i}\right) \in$ $R^{m \times m}, i=1,2, \ldots, n$, represent the known control coefficient matrices, and $h_{i}\left(\bar{x}_{i}\left(t-\tau_{i}\right)\right) \in R^{m \times m}, i=1,2, \ldots, n$, indicate the known time-delay functions. $\Delta f_{i}\left(\bar{x}_{i}(t)\right) \in R^{m}, i=1,2, \ldots, n$, denote the unknown nonlinear functions which contain both parametric and nonparametric uncertainties. $\tau_{i} \in R, i=$ $1,2, \ldots, n$, stand for constant unknown time delays. $d_{i}(t) \in$ $R^{m}, i=1,2, \ldots, n$, mean the unknown external disturbance. In this paper, we impose the following assumptions and lemmas.

Assumption 1 (see [5]). The ideal tracking signals $y_{r}(t)$ and their derivatives $\dot{y}_{r}(t)$ are known and continuous.

Assumption 2 (see [11]). The external disturbance $d_{i}(t)$ means the slow varying signal, while it is restricted in the bound of $\left\|\dot{d}_{i}(t)\right\| \leq \bar{d}_{i}, \bar{d}_{i}>0$.

Assumption 3 (see [10]). For $i=1, \ldots, n$, the known continuous function $g_{i}\left(\bar{x}_{i}(t)\right)$ satisfies $g_{i}\left(\bar{x}_{i}(t)\right) \neq 0$.

Lemma 4 (see [9]). Consider a class of nonlinear systems $\dot{x}=$ $f(x)$. For any initial conditions $x(0) \in R^{n}$, if there exists a $C^{1}$ continuous and positive definite Lyapunov function satisfying $\omega_{1}(x) \leq V(x) \leq \omega_{2}(x), \omega_{i}(\|x\|): R^{n} \rightarrow R, i=1,2$, mean the $K$ class functions, such that $\dot{V} \leq-c_{1} V(x)+c_{2}, c_{1}>0$, $c_{2}>0$, then one can conclude that the solution $x(t)$ is uniformly bounded.

Lemma 5 (see [3]). For $v>0$, if there exists one set $Z$ defined by $Z:=\{z|| z \mid \leq 0.8814 v\}$, then one can conclude that, for any $z \notin Z$, the inequality $1-2 \tanh ^{2}(z / v)<0$ is satisfied.

Lemma 6 (see [10]). For any continuous function $f(z)$, there exists a valid linear neural network to approximate it by choosing enough nodes on the compact set $z \in N_{z}$. The basic function can be selected as Gaussian function $s_{i}(z)=$ $e^{-\left\|z-\mu_{i}\right\|^{2} / c_{i}}$, where $c_{i}>0$ is the width of function and $\mu_{i} \in R$ represent the center of function. The neural network approximator consists of the weight estimate vector $\widehat{W}(z)$ and Gaussian function $S(z)=\left[s_{1}(z), \ldots, s_{n}(z)\right]^{T}$, which can be written as $f_{p}(z)=\widehat{W}^{T}(z) S(z)+\varepsilon$. Assume that $W^{*}(z)$ means the optimal approximating weight, such that

$$
W^{*}(z):=\arg \min _{\widehat{W} \in R^{n}}\left\{\sup _{z \in \Omega_{z}}\left\|f(z)-f_{p}(z)\right\|\right\} .
$$

In addition, the optimal approximator of continuous function $f(z)$ can be written as 


$$
f(z)=W^{* T}(z) S(z)+\varepsilon^{*},
$$

where $\varepsilon^{*}$ indicates the optimal approximate error.

\section{Controller Design and Stability Analysis}

In this section, the objective is to propose a robust prescribed performance control law for uncertain nonlinear systems such that the closed-loop errors converge to a small neighborhood of the origin.

\subsection{Prescribed Performance Controller Design}

Step 1. Define the tracking errors $\widetilde{z}_{1}(t) \in R^{m}$ and $z_{2}(t) \in R^{m}$ as follows:

$$
\begin{aligned}
& \widetilde{z}_{1}(t)=x_{1}(t)-y_{d}(t) \\
& z_{2}(t)=x_{2}(t)-x_{2}^{*}(t),
\end{aligned}
$$

where $y_{d}(t) \in R^{m}$ is the ideal tracking signal and $x_{2}^{*}(t) \in R^{m}$ is the immediate control. The output error transformation can be defined in the form of [18]

$$
\begin{aligned}
z_{1 j}(t) & =Q^{-1}\left(\frac{\widetilde{z}_{1 j}(t)}{\rho_{j}(t)}, \bar{\alpha}_{j}(t), \underline{\alpha}_{j}(t)\right) \\
& =\frac{1}{2} \ln \frac{\underline{\alpha}_{j}+\left(\widetilde{z}_{1 j}(t) / \rho_{j}(t)\right)}{\bar{\alpha}_{j}-\left(\widetilde{z}_{1 j}(t) / \rho_{j}(t)\right)}, \quad j=1, \ldots, m,
\end{aligned}
$$

where $\bar{\alpha}_{j}$ and $\underline{\alpha}_{j}$ are the positive constants and $\rho_{j}(t)$ indicates the performance function, which can be chosen as [26]

$$
\rho_{j}(t)=\left(\rho_{j 0}-\rho_{j \infty}\right) e^{-l t}+\rho_{j \infty} .
$$

The constant $\rho_{j \infty}>0$ is the maximum amplitude of the tracking error at the steady state. The decreasing rate $e^{-l_{j} t}$ of $\rho_{j}(t)$ represents the desired convergence speed of the tracking error. Therefore, the appropriate choice of the performance function $\rho_{j}(t)$ and the design constant imposes bounds on the system output trajectory.

Define $f_{1}=\left[f_{11}, \ldots, f_{1 m}\right]^{T} \in R^{m}$ and $g_{1}=\left[g_{11}^{T}\right.$, $\left.\ldots, g_{1 m}^{T}\right]^{T} \in R^{m \times m}$. Thus, the time derivative of $z_{1 j}(t)$ becomes

$$
\begin{aligned}
\dot{z}_{1 j}(t)= & \frac{\partial z_{1 j}(t)}{\partial\left(\widetilde{z}_{1 j}(t) / \rho_{j}(t)\right)} \frac{1}{\rho_{j}(t)} \dot{\tilde{z}}_{1 j}(t)-\frac{\partial z_{1 j}(t)}{\partial\left(\widetilde{z}_{1 j}(t) / \rho_{j}(t)\right)} \frac{\widetilde{z}_{1 j}(t)}{\rho_{j}^{2}(t)} \dot{\rho}_{j}(t) \\
= & \frac{1}{2} \frac{\bar{\alpha}_{j}+\underline{\alpha}_{j}}{\left(\underline{\alpha}_{j}+\left(\widetilde{z}_{1 j}(t) / \rho_{j}(t)\right)\right)\left(\bar{\alpha}_{j}-\left(\widetilde{z}_{1 j}(t) / \rho_{j}(t)\right)\right)}\left(\frac{1}{\rho_{j}(t)} \dot{\tilde{z}}_{1 j}(t)-\frac{\widetilde{z}_{1 j}(t)}{\rho_{j}^{2}(t)} \dot{\rho}_{j}(t)\right) \\
= & \frac{1}{2} \frac{\bar{\alpha}_{j}+\underline{\alpha}_{j}}{\left(\underline{\alpha}_{j}+\left(\widetilde{z}_{1 j}(t) / \rho_{j}(t)\right)\right)\left(\bar{\alpha}_{j}-\left(\widetilde{z}_{1 j}(t) / \rho_{j}(t)\right)\right)} \\
& \cdot\left(-\frac{\widetilde{z}_{1 j}(t)}{\rho_{j}^{2}(t)} \dot{\rho}_{j}(t)+\frac{f_{1 j}\left(x_{1}(t)\right)+g_{1 j}\left(x_{1}(t)\right) x_{2}(t)+h_{1 j}\left(x_{1}\left(t-\tau_{1}\right)\right)+\Delta f_{1 j}\left(x_{1}(t)\right)+d_{1 j}(t)-\dot{y}_{d j}(t)}{\rho_{j}(t)}\right)
\end{aligned}
$$

In order to simplify the analysis, we define $M_{1 j}$ and $N_{1 j}$ as follows:

$$
\begin{aligned}
& M_{1 j}(t) \\
& \quad=\frac{\bar{\alpha}_{j}+\underline{\alpha}_{j}}{2 \rho_{j}\left(\underline{\alpha}_{j}+\left(\widetilde{z}_{1 j}(t) / \rho_{j}(t)\right)\right)\left(\bar{\alpha}_{j}-\left(\widetilde{z}_{1 j}(t) / \rho_{j}(t)\right)\right)} \\
& \quad>0 . \\
& N_{1 j}(t) \\
& \quad=\frac{\bar{\alpha}_{j}+\underline{\alpha}_{j}}{2 \rho_{j}^{2}(t)\left(\underline{\alpha}_{j}+\left(\widetilde{z}_{1 j}(t) / \rho_{j}(t)\right)\right)\left(\bar{\alpha}_{j}-\left(\widetilde{z}_{1 j}(t) / \rho_{j}(t)\right)\right)} \\
& \cdot \widetilde{z}_{1 j}(t) \dot{\rho}_{j}(t)
\end{aligned}
$$

Furthermore, we can define $M_{1}(t)=\operatorname{diag}\left\{M_{11}(t), \ldots\right.$, $\left.M_{1 m}(t)\right\}$ and $N_{1}(t)=\left[N_{11}(t), \ldots, N_{1 m}(t)\right]^{T}$, then we have

$$
\begin{aligned}
& \dot{z}_{1}(t)=M_{1}(t) \dot{\vec{z}}_{1}(t)-N_{1}(t)=M_{1}(t)\left(f_{1}\left(x_{1}(t)\right)\right. \\
& +g_{1}\left(x_{1}(t)\right) x_{2}(t)-\dot{y}_{d}(t)+\Delta f_{1}\left(x_{1}(t)\right) \\
& \left.+h_{1}\left(x_{1}\left(t-\tau_{1}\right)\right)+d_{1}(t)\right)-N_{1}(t) .
\end{aligned}
$$

Since $\Delta f_{1}\left(x_{1}(t)\right)$ is unknown, using the RBFNN to approximate it, we obtain

$$
M_{1}(t) \Delta f_{1}\left(x_{1}(t)\right)+\gamma_{1}(t)=\eta_{1}^{-1} W_{1}^{* T} S_{1}+\varepsilon_{1}^{*}
$$

where $\gamma_{1}(t)=\left[\gamma_{1,1}(t), \ldots, \gamma_{1, m}(t)\right]^{T}$, and

$$
\gamma_{1, j}(t)=\frac{16}{\widetilde{z}_{1 j}(t)} \tanh ^{2}\left(\frac{\widetilde{z}_{1 j}(t)}{v_{1}}\right) h_{1 j}^{2}\left(x_{1}(t)\right),
$$


Substituting (10) into (1) results in

$$
\begin{aligned}
\dot{x}_{1}(t)= & f_{1}\left(x_{1}(t)\right)+g_{1}\left(x_{1}(t)\right) x_{2}(t) \\
& +h_{1}\left(x_{1}\left(t-\tau_{1}\right)\right)-M_{1}^{-1}(t) \gamma_{1}(t) \\
& +\eta_{1}^{-1} M_{1}^{-1}(t) W_{1}^{* T} S_{1}+\chi_{1}(t) .
\end{aligned}
$$

Invoking (4), (9), and (10), the time derivative of $z_{1}(t)$ can be rewritten as

$$
\begin{aligned}
& \dot{z}_{1}(t)=M_{1}(t) \\
& \quad \cdot\left(f_{1}\left(x_{1}(t)\right)+g_{1}\left(x_{1}(t)\right) z_{2}(t)+g_{1}\left(x_{1}(t)\right) x_{2}^{*}(t)\right. \\
& \left.+h_{1}\left(x_{1}\left(t-\tau_{1}\right)\right)-\dot{y}_{d}(t)\right)-\gamma_{1}(t)+\eta_{1}^{-1} W_{1}^{* T} S_{1} \\
& \quad+M_{1}(t) \chi_{1}(t)-N_{1}(t) .
\end{aligned}
$$

Construct updating law of the RBFNN

$$
\dot{\widehat{W}}_{1}=\Gamma_{1}\left(\eta_{1}^{-1} z_{1}(t) S_{1}-\sigma_{1} \Gamma_{1}^{-1} \widehat{W}_{1}\right),
$$

where $\sigma_{1}>0$ is a design parameter. Furthermore, the DOB can be chosen as

$$
\begin{aligned}
& \widehat{\chi}_{1}(t)=w_{1}(t)+\eta_{1} x_{1}(t) \\
& \dot{w}_{1}(t)=-\eta_{1} w_{1}(t)-\eta_{1}\left(f_{1}\left(x_{1}(t)\right)+g_{1}\left(x_{1}(t)\right) x_{2}(t)\right. \\
& \left.\quad+\eta_{1}^{-1} \widehat{W}_{1}^{T} S_{1}+\eta_{1} x_{1}(t)\right)+M_{1}^{T}(t) z_{1}(t) .
\end{aligned}
$$

According to (15), we obtain

$$
\dot{\hat{\chi}}_{1}(t)=\eta_{1} \widetilde{\chi}_{1}(t)+M_{1}^{T}(t) z_{1}(t)+\widetilde{W}_{1}^{T} S_{1} .
$$

According to the neural network updating law (14) and DOB (15), the immediate control is chosen

$$
\begin{aligned}
& x_{2}^{*}(t)=g_{1}^{-1}\left(x_{1}(t)\right)\left(-M_{1}^{-1}(t) K_{1} z_{1}(t)-f_{1}\left(x_{1}(t)\right)\right. \\
& \quad+\dot{y}_{d}(t)+M_{1}^{-1}(t) N_{1}(t)-M_{1}^{-1}(t) \gamma_{1}(t) \\
& \left.-\eta_{1}^{-1} \widehat{W}_{1}^{T} S_{1}-\widehat{\chi}_{1}(t)\right),
\end{aligned}
$$

where $M_{1}(t)=\operatorname{diag}\left\{M_{11}(t), \ldots, M_{1 m}(t)\right\} . \dot{y}_{d}(t)$ is the time derivative of reference trajectory, and $K_{1} \in R^{m \times m}$ is the constant positive definite matrices. $\widehat{W}_{1}$ is the estimated values of $W_{1}^{*}, \widetilde{W}_{1}$ represents the estimated error, and $\widetilde{W}_{1}=W_{1}^{*}-$ $\widehat{W}_{1}$. Substituting (17) into (13), the time derivative of $z_{1}(t)$ becomes

$$
\begin{aligned}
\dot{z}_{1}(t)= & -K_{1} z_{1}(t)+M_{1}(t) g_{1}\left(x_{1}(t)\right) z_{2}(t)-\gamma(t) \\
& +M_{1}(t) h_{1}\left(x_{1}\left(t-\tau_{1}\right)\right)+\eta_{1}^{-1} \widetilde{W}_{1}^{T} S_{1} \\
& +M_{1}(t) \widetilde{\chi}_{1}(t) .
\end{aligned}
$$

Choose the Lyapunov-Krasovskii functional candidate as

$$
\begin{aligned}
V_{1}= & \frac{z_{1}^{T}(t) z_{1}(t)}{2}+\int_{t-\tau_{1}}^{t} P_{1}\left(x_{1}(\tau)\right) d \tau \\
& +\operatorname{tr}\left(\widetilde{W}_{1}^{T} \Gamma_{1}^{-1} \widetilde{W}_{1}\right)+\frac{1}{2} \widetilde{\chi}_{1}^{T}(t) \widetilde{\chi}_{1}(t),
\end{aligned}
$$

where the positive function $P_{1}\left(x_{1}(t)\right)$ can be designed as follows:

$$
P_{1}\left(x_{1}(t)\right)=\frac{h_{1}^{T}\left(x_{1}(t)\right) h_{1}\left(x_{1}(t)\right)}{2} .
$$

Then the time derivative of $V_{1}$ is

$$
\begin{aligned}
\dot{V}_{1} & =z_{1}^{T}(t)\left(-K_{1} z_{1}(t)+M_{1}(t) g_{1}\left(x_{1}(t)\right) z_{2}(t)\right. \\
& +M_{1}(t) h_{1}\left(x_{1}\left(t-\tau_{1}\right)\right)-\gamma_{1}(t)+\eta_{1}^{-1} \widetilde{W}_{1}^{T} S_{1} \\
& \left.+M_{1}(t) \chi_{1}(t)\right)+P_{1}\left(x_{1}(t)\right)-P_{1}\left(x_{1}\left(t-\tau_{1}\right)\right) \\
& -\operatorname{tr}\left(\widetilde{W}_{1}^{T} \Gamma_{1}^{-1} \dot{\widehat{W}}_{1}\right)-\widetilde{\chi}_{1}^{T}(t) \dot{\bar{\chi}}_{1}(t)+\widetilde{\chi}_{1}^{T}(t) \dot{\chi}_{1}(t) \\
& =-z_{1}^{T}(t) K_{1} z_{1}(t)+z_{1}^{T}(t) \widetilde{W}_{1}^{T} S_{1}+z_{1}^{T}(t) \\
& \cdot h_{1}\left(x_{1}\left(t-\tau_{1}\right)\right)-z_{1}^{T}(t) \gamma_{1}(t)+z_{1}^{T}(t) M_{1}(t) \\
& \cdot g_{1}\left(x_{1}(t)\right) z_{2}(t)+\eta_{1}^{-1} z_{1}^{T}(t) \widetilde{W}_{1}^{T} S_{1}+z_{1}^{T}(t) M_{1}(t) \\
& \cdot \widetilde{\chi}_{1}(t)+P_{1}\left(x_{1}(t)\right)-P_{1}\left(x_{1}\left(t-\tau_{1}\right)\right) \\
& -\operatorname{tr}\left(\widetilde{W}_{1}^{T} \Gamma_{1}^{-1} \dot{\widehat{W}}_{1}\right)-\widetilde{\chi}_{1}^{T}(t) \dot{\hat{\chi}}_{1}(t)+\widetilde{\chi}_{1}^{T}(t) \dot{\chi}_{1}(t) .
\end{aligned}
$$

Substituting (14) into (21), we obtain

$$
\begin{aligned}
\dot{V}_{1}= & -z_{1}^{T}(t) K_{1} z_{1}(t)+z_{1}^{T}(t) \widetilde{W}_{1}^{T} S_{1} \\
& +z_{1}^{T}(t) h_{1}\left(x_{1}\left(t-\tau_{1}\right)\right) \\
& +z_{1}^{T}(t) M_{1}(t) g_{1}\left(x_{1}(t)\right) z_{2}(t)-z_{1}^{T}(t) \gamma_{1}(t) \\
& +z_{1}^{T}(t) M_{1}(t) \widetilde{\chi}_{1}+P_{1}\left(x_{1}(t)\right) \\
& -P_{1}\left(x_{1}\left(t-\tau_{1}\right)\right)+\sigma_{1} t r\left(\widetilde{W}_{1}^{T} \Gamma_{1}^{-1} \widehat{W}_{1}\right) \\
& -\widetilde{\chi}_{1}^{T}(t) \dot{\hat{\chi}}_{1}(t)+\tilde{\chi}_{1}^{T}(t) \dot{\chi}_{1}(t) .
\end{aligned}
$$

Substituting (16) into (22) yields

$$
\begin{aligned}
\dot{V}_{1}= & -z_{1}^{T}(t) K_{1} z_{1}(t)+z_{1}^{T}(t) \widetilde{W}_{1}^{T} S_{1} \\
& +z_{1}^{T}(t) h_{1}\left(x_{1}\left(t-\tau_{1}\right)\right)-z_{1}^{T}(t) \gamma_{1}(t) \\
& +z_{1}^{T}(t) M_{1}(t) g_{1}\left(x_{1}(t)\right) z_{2}(t)+P_{1}\left(x_{1}(t)\right) \\
& -P_{1}\left(x_{1}\left(t-\tau_{1}\right)\right)+\sigma_{1} t r\left(\widetilde{W}_{1}^{T} \Gamma_{1}^{-1} \widehat{W}_{1}\right) \\
& -\eta_{1} \widetilde{\chi}_{1}^{T}(t) \widetilde{\chi}_{1}(t)-\widetilde{\chi}_{1}^{T}(t) \widetilde{W}_{1}^{T} S_{1}+\widetilde{\chi}_{1}^{T}(t) \dot{\chi}_{1}(t) .
\end{aligned}
$$

Step $i$. Define the error variables $z_{i}(t) \in R^{m}$ and $z_{i+1}(t) \in R^{m}$

$$
\begin{aligned}
z_{i}(t) & =x_{i}(t)-x_{i}^{*}(t) \\
z_{i+1}(t) & =x_{i+1}(t)-x_{i+1}^{*}(t),
\end{aligned}
$$

where $x_{i}^{*}(t)$ is a virtual control law. Combining (1) and (24) and differentiating $z_{i}(t)$ with respect to time, we have

$$
\begin{aligned}
\dot{z}_{i}(t)= & f_{i}\left(\bar{x}_{i}(t)\right)+g_{i}\left(\bar{x}_{i}(t)\right) x_{i+1}(t)+h_{i}\left(\bar{x}_{i}\left(t-\tau_{i}\right)\right) \\
& +\Delta f_{i}\left(\bar{x}_{i}(t)\right)+d_{i}(t)-\dot{x}_{i}^{*}(t) .
\end{aligned}
$$


Since $\Delta f_{i}\left(\bar{x}_{i}(t)\right)$ is unknown, we use RBFNN to approximate uncertain function. $\widehat{W}_{i}$ indicates the estimate of $W_{i}^{*}$, and $\varepsilon_{i}^{*}$ is the approximate error. $\widetilde{W}_{i}$ represents the estimated error, which is defined as $\widetilde{W}_{i}=W_{i}^{*}-\widehat{W}_{i}$. Define the variable $\chi_{i}(t)=$ $\varepsilon_{i}^{*}+d_{i}(t)$.

$$
\Delta f_{i}\left(\bar{x}_{i}(t)\right)+\gamma_{i}(t)=\eta_{i}^{-1} W_{i}^{* T} S_{i}+\varepsilon_{i}^{*},
$$

where $\gamma_{i}(t)=\left[\gamma_{i, 1}(t), \ldots, \gamma_{i, m}(t)\right]^{T}$, and

$$
\gamma_{i, j}(t)=\frac{16}{z_{i j}(t)} \tanh ^{2}\left(\frac{z_{i j}(t)}{v_{i}}\right) h_{i j}^{2}\left(\bar{x}_{i}(t)\right),
$$

$$
j=1, \ldots, m \text {. }
$$

Substituting (26) into (1) yields

$$
\begin{aligned}
\dot{x}_{i}(t)= & f_{i}\left(\bar{x}_{i}(t)\right)+g_{i}\left(\bar{x}_{i}(t)\right) x_{i+1}(t) \\
& +h_{i}\left(\bar{x}_{i}\left(t-\tau_{i}\right)\right)-\gamma_{i}(t)+\eta_{i}^{-1} W_{i}^{* T} S_{i}+\chi_{i}(t) .
\end{aligned}
$$

Moreover, substituting (26) into (25), we have

$$
\begin{aligned}
\dot{z}_{i}(t)= & f_{i}\left(\bar{x}_{i}(t)\right)+g_{i}\left(\bar{x}_{i}(t)\right) x_{i+1}(t) \\
& +h_{i}\left(\bar{x}_{i}\left(t-\tau_{i}\right)\right)-\gamma_{i}(t)+\eta_{i}^{-1} W_{i}^{* T} S_{i}+\chi_{i}(t) \\
& -\dot{x}_{i}^{*}(t) .
\end{aligned}
$$

Construct updating law of the RBFNN

$$
\dot{\bar{W}}_{i}=\Gamma_{i}\left(\eta_{1}^{-1} z_{i}(t) S_{i}-\sigma_{i} \Gamma_{i}^{-1} \widehat{W}_{i}\right),
$$

where $\sigma_{i}>0$ is a design parameter. Furthermore, the DOB can be chosen as

$$
\begin{aligned}
& \widehat{\chi}_{i}(t)=w_{i}(t)+\eta_{i} x_{i}(t) \\
& \dot{w}_{i}(t)=-\eta_{i} w_{i}(t)-\eta_{i}\left(f_{i}\left(\bar{x}_{i}(t)\right)+g_{i}\left(\bar{x}_{i}(t)\right) x_{i+1}\right. \\
&\left.\quad+\eta_{i}^{-1} \widehat{W}_{i}^{T} S_{i}+\eta_{i} x_{i}(t)\right)+z_{i}(t) .
\end{aligned}
$$

According to (31), we obtain

$$
\dot{\bar{\chi}}_{i}(t)=\eta_{i} \widetilde{\chi}_{i}(t)+z_{i}(t)+\widetilde{W}_{i}^{T} S_{i} .
$$

Hence, the virtual control law $x_{i+1}^{*}(t)$ is proposed as

$$
\begin{aligned}
& x_{i+1}^{*}(t)=g_{i}^{-1}\left(\bar{x}_{i}(t)\right)\left(-K_{i} z_{i}(t)\right. \\
& \quad-g_{i-1}^{T}\left(\bar{x}_{i-1}(t)\right) z_{i-1}(t)-f_{i}\left(\bar{x}_{i}(t)\right)+\dot{x}_{i}^{*}(t) \\
& \left.\quad-\eta_{i}^{-1} \widehat{W}_{i}^{T} S_{i}-\widehat{\chi}_{i}(t)\right) .
\end{aligned}
$$

Choose the Lyapunov-Krasovskii functional candidate as

$$
\begin{aligned}
V_{i}= & \frac{1}{2} z_{i}^{T}(t) z_{i}(t)+\int_{t-\tau_{i}}^{t} P_{i}\left(\bar{x}_{i}(\tau)\right) d \tau \\
& +\operatorname{tr}\left(\widetilde{W}_{i}^{T} \Gamma_{i}^{-1} \widetilde{W}_{i}\right)+\frac{1}{2} \widetilde{\chi}_{i}^{T}(t) \widetilde{\chi}_{i}(t),
\end{aligned}
$$

where the positive function $P_{i}\left(\bar{x}_{i}(\tau)\right)$ is given in the form of

$$
P_{i}\left(\bar{x}_{i}(\tau)\right)=\frac{1}{2} h_{i}^{T}\left(\bar{x}_{i}(\tau)\right) h_{i}\left(\bar{x}_{i}(\tau)\right) .
$$

Similar to Step 1, the time derivative of $\dot{V}_{i}$ can be rewritten as

$$
\begin{aligned}
\dot{V}_{i}= & -z_{i}^{T}(t) K_{i} z_{i}(t)+z_{i}^{T}(t) g_{i}\left(\bar{x}_{i}(t)\right) z_{i+1}(t) \\
& -z_{i}^{T}(t) g_{i-1}^{T}\left(\bar{x}_{i-1}(t)\right) z_{i-1}(t)-z_{i}^{T}(t) \gamma_{i}(t) \\
& +z_{i}^{T}(t) h_{i}\left(\bar{x}_{i}\left(t-\tau_{i}\right)\right)+P_{i}\left(\bar{x}_{i}(t)\right) \\
& -P_{i}\left(\bar{x}_{i}\left(t-\tau_{i}\right)\right)+\sigma_{i} t r\left(\widetilde{W}_{i}^{T} \Gamma_{i}^{-1} \widehat{W}_{i}\right) \\
& -\eta_{i} \widetilde{\chi}_{i}^{T}(t) \widetilde{\chi}_{i}(t)-\widetilde{\chi}_{i}^{T}(t) \widetilde{W}_{i}^{T} S_{i}+\widetilde{\chi}_{i}^{T}(t) \dot{\chi}_{i}(t) .
\end{aligned}
$$

Step $n$. Define the error variable $z_{n}(t) \in R^{m}$ :

$$
z_{n}(t)=x_{n}(t)-x_{n}^{*}(t)
$$

Invoking (1) and (37), differentiating $z_{n}(t)$ with respect to time yields

$$
\begin{aligned}
\dot{z}_{n}(t)= & f_{n}\left(\bar{x}_{n}(t)\right)+g_{n}\left(\bar{x}_{n}(t)\right) u(t)+h_{n}\left(\bar{x}_{n}(t-\tau)\right) \\
& +\Delta f_{n}\left(\bar{x}_{n}(t)\right)-\dot{x}_{n}^{*}(t) .
\end{aligned}
$$

Since $\Delta f_{n}\left(\bar{x}_{n}(t)\right)$ is unknown, the RBFNN is used to approximate $\Delta f_{n}\left(\bar{x}_{n}(t)\right) . \widehat{W}_{n}$ indicates the estimate of $W_{n}^{*}$, and $\varepsilon_{n}^{*}$ is the approximate error. $\widetilde{W}_{n}$ represents the estimated error, which is defined as $\widetilde{W}_{n}=W_{n}^{*}-\widehat{W}_{n}$. Define the variable $\chi_{n}=\varepsilon_{n}^{*}+d_{n}(t)$.

$$
\Delta f_{n}\left(\bar{x}_{n}(t)\right)+\gamma_{n}(t)=\eta_{n}^{-1} W_{n}^{* T} S_{n}+\varepsilon_{n}^{*},
$$

where $\gamma_{n}(t)=\left[\gamma_{n, 1}(t), \ldots, \gamma_{n, m}(t)\right]^{T}$, and

$$
\begin{aligned}
\gamma_{n, j}(t)=\frac{16}{z_{n j}(t)} \tanh ^{2}\left(\frac{z_{n j}(t)}{v_{n}}\right) h_{n j}^{2}\left(\bar{x}_{n}(t)\right), & \\
& j=1, \ldots, m .
\end{aligned}
$$

Substituting (39) into (1) yields

$$
\begin{aligned}
\dot{x}_{n}(t)= & f_{n}\left(\bar{x}_{n}(t)\right)+g_{n}\left(\bar{x}_{n}(t)\right) u(t) \\
& +h_{n}\left(\bar{x}_{n}\left(t-\tau_{n}\right)\right)-\gamma_{n}(t)+\eta_{n}^{-1} W_{n}^{* T} S_{n} \\
& +\chi_{n}(t) .
\end{aligned}
$$

Invoking (38) and (39), one obtains

$$
\begin{aligned}
\dot{z}_{n}(t)= & f_{n}\left(\bar{x}_{n}(t)\right)+g_{n}\left(\bar{x}_{n}(t)\right) u(t) \\
& +h_{n}\left(\bar{x}_{n}\left(t-\tau_{n}\right)\right)-\gamma_{n}(t)+\eta_{n}^{-1} W_{n}^{* T} S_{n} \\
& +\chi_{n}(t)-\dot{x}_{n}^{*}(t) .
\end{aligned}
$$

Construct updating law of the RBFNN

$$
\dot{\widehat{W}}_{n}(t)=\Gamma_{n}\left(\eta_{n}^{-1} z_{n}(t) S_{n}-\sigma_{n} \Gamma_{n}^{-1} \widehat{W}_{n}(t)\right),
$$


where $\sigma_{n} \in R, \sigma_{n}>0$, is a design parameter. Furthermore, the $\mathrm{DOB}$ can be chosen as

$$
\begin{aligned}
& \widehat{\chi}_{n}(t)=w_{n}(t)+\eta_{n} x_{n}(t) \\
& \dot{w}_{n}(t)=-\eta_{n} w_{n}(t)-\eta_{n}\left(f_{n}\left(\bar{x}_{n}(t)\right)+g_{n}\left(\bar{x}_{n}(t)\right) u(t)\right. \\
& \left.\quad+\eta_{n}^{-1} \widehat{W}_{n}^{T} S_{n}+\eta_{n} x_{n}(t)\right)+z_{n}(t) .
\end{aligned}
$$

According to (44), we obtain

$$
\dot{\hat{\chi}}_{n}(t)=\eta_{n} \widetilde{\chi}_{n}(t)+z_{n}(t)+\widetilde{W}_{n}^{T} S_{n}
$$

Therefore, design the control law $u(t)$ as

$$
\begin{aligned}
u(t) & =g_{n}^{-1}\left(\bar{x}_{n}(t)\right)\left(-K_{n} z_{n}(t)\right. \\
& -g_{n-1}^{T}\left(\bar{x}_{n-1}(t)\right) z_{n-1}(t)-f_{n}\left(\bar{x}_{n}(t)\right)+\dot{x}_{n}^{*}(t) \\
& \left.-\eta_{n}^{-1} \widehat{W}_{n}^{T} S_{n}-\widehat{\chi}_{n}(t)\right)
\end{aligned}
$$

where $K_{n} \in R^{m \times m}, K_{n}>0$ is design matrix. Choose the Lyapunov-Krasovskii functional candidate as

$$
\begin{aligned}
V_{n}= & \frac{1}{2} z_{n}^{T}(t) z_{n}(t)+\int_{t-\tau_{n}}^{t} P_{n}\left(\bar{x}_{n}(\tau)\right) d \tau \\
& +\operatorname{tr}\left(\widetilde{W}_{n}^{T} \Gamma_{n}^{-1} \widetilde{W}_{n}\right)+\frac{1}{2} \widetilde{\chi}_{n}^{T}(t) \widetilde{\chi}_{n}(t)
\end{aligned}
$$

and the positive function $P_{n}\left(\bar{x}_{n}(\tau)\right)$ can be designed as follows:

$$
P_{n}\left(\bar{x}_{n}(\tau)\right)=\frac{1}{2} h_{n}^{T}\left(\bar{x}_{n}(\tau)\right) h_{n}\left(\bar{x}_{n}(\tau)\right)
$$

According to the derivatives in Step 1 and Step i, we have

$$
\begin{aligned}
\dot{V}_{n}= & -z_{n}^{T}(t) K_{n} z_{n}(t)-z_{n}^{T}(t) g_{n-1}^{T}\left(\bar{x}_{n-1}(t)\right) z_{n-1}(t) \\
& +z_{n}^{T}(t) h_{n}\left(\bar{x}_{n}\left(t-\tau_{n}\right)\right)-z_{n}^{T}(t) \gamma_{n}(t) \\
& +P_{n}\left(\bar{x}_{n}(t)\right)-P_{n}\left(\bar{x}_{n}\left(t-\tau_{n}\right)\right) \\
& -\sigma_{n} \operatorname{tr}\left(\widetilde{W}_{n}^{T} \Gamma_{n}^{-1} \widehat{W}_{n}\right)-\eta_{n} \widetilde{\chi}_{n}^{T}(t) \widetilde{\chi}_{n}(t) \\
& -\widetilde{\chi}_{n}^{T}(t) \widetilde{W}_{n}^{T} S_{n}+\widetilde{\chi}_{n}^{T}(t) \dot{\chi}_{n}(t) .
\end{aligned}
$$

From the above inductive design procedure, moreover, we can conclude the following theorem.

\subsection{Stability Analysis}

Theorem 7. Considering the system dynamics described by (1), under the adaptive neural updated laws (14), (30), and (43), the disturbance observers (15), (31), and (45), and the prescribed performance-based control laws (17), (33), and (46), we can conclude that the trajectories of the closed-loop system are semiglobally uniformly bounded, while the tracking error $\widetilde{z}_{1}(t)$ converges to a compact set $Q_{z}$ asymptotically, where $C$ and $\kappa$ are defined in (50).

$$
\begin{gathered}
\kappa=\min \left\{\left(\lambda_{\min }\left(K_{i}\right)-\frac{\vartheta_{\xi i}+1}{2}\right),\right. \\
\left.\left(\eta_{i}-\frac{1}{2 \vartheta_{\xi i}}-\frac{\delta_{S} \varphi_{i}}{2}-\frac{\pi_{i}}{2}\right), \frac{\sigma_{i}}{2}\right\} \\
C=\sum_{i=1}^{n} \frac{\sigma_{i}}{2} \lambda_{\min }\left(\Gamma_{i}^{-1}\right)\left\|W_{i}^{*}\right\|^{2}+\sum_{i=1}^{n} \frac{1}{2 \pi_{i}}\left\|\dot{\chi}_{i}(t)\right\|^{2},
\end{gathered}
$$

where $Q_{z}$ can be made as small as desired by appropriately choosing design parameters. $\lambda_{\text {min }}$ represents the minimum eigenvalue of the matrix. $\lambda_{\min }$ represents the minimum eigenvalue of the matrix.

Proof. For analytical purposes, define the total Lyapunov function

$$
V_{\Xi}=\sum_{i=1}^{n} V_{i},
$$

where definition of $V_{i}$ can be referred to (19), (34), and (47). According to (23), (36), and (49), we obtain

$$
\begin{aligned}
\dot{V}_{\Xi}= & -\sum_{i=1}^{n} z_{i}^{T}(t) K_{i} z_{i}(t)-\sum_{i=1}^{n} \eta_{i} \widetilde{\chi}_{i}^{T}(t) \widetilde{\chi}_{i}(t) \\
& +\sum_{i=1}^{n} z_{i}^{T}(t) h_{i}\left(\bar{x}_{i}\left(t-\tau_{i}\right)\right)+\sum_{i=1}^{n} z_{i}^{T}(t) \widetilde{\chi}_{i}(t) \\
& -\sum_{i=1}^{n} z_{i}^{T}(t) \gamma_{i}(t)-\sum_{i=1}^{n} \widetilde{\chi}_{i}^{T}(t) \widetilde{W}_{i}^{T} S_{i} \\
& +\sum_{i=1}^{n} \widetilde{\chi}_{i}^{T}(t) \dot{\chi}_{i}(t) \\
& +\sum_{i=1}^{n}\left(P_{i}\left(\bar{x}_{i}(t)\right)-P_{i}\left(\bar{x}_{i}\left(t-\tau_{i}\right)\right)\right) \\
& -\sum_{i=1}^{n} \sigma_{i} t r\left(\widetilde{W}_{i}^{T} \Gamma_{i}^{-1} \widehat{W}_{i}\right) .
\end{aligned}
$$

Considering the fact that

$$
\begin{aligned}
& z_{i}^{T}(t) h_{i}\left(\bar{x}_{i}\left(t-\tau_{i}\right)\right) \\
& \quad \leq \frac{1}{2} z_{i}^{T}(t) z_{i}(t)+\frac{1}{2} h_{i}^{T}\left(\bar{x}_{i}\left(t-\tau_{i}\right)\right) h_{i}\left(\bar{x}_{i}\left(t-\tau_{i}\right)\right)
\end{aligned}
$$


and $P_{i}\left(\bar{x}_{i}\left(t-\tau_{i}\right)\right)=(1 / 2) h_{i}^{T}\left(\bar{x}_{i}\left(t-\tau_{i}\right)\right) h_{i}\left(\bar{x}_{i}\left(t-\tau_{i}\right)\right)$, we have

$$
\begin{aligned}
\dot{V}_{\Xi} \leq & -\sum_{i=1}^{n} z_{i}^{T}(t) K_{i} z_{i}(t)-\sum_{i=1}^{n} \eta_{i} \widetilde{\chi}_{i}^{T}(t) \widetilde{\chi}_{i}(t) \\
& +\sum_{i=1}^{n} z_{i}^{T}(t) \widetilde{\chi}_{i}(t)-\sum_{i=1}^{n} z_{i}^{T}(t) \gamma_{i}(t) \\
& -\sum_{i=1}^{n} \widetilde{\chi}_{i}^{T}(t) \widetilde{W}_{i}^{T} S_{i}+\sum_{i=1}^{n} \tilde{\chi}_{i}^{T}(t) \dot{\chi}_{i}(t) \\
& +\frac{1}{2} \sum_{i=1}^{n} z_{i}^{T}(t) z_{i}(t)+\sum_{i=1}^{n} P_{i}\left(\bar{x}_{i}(t)\right) \\
& -\sum_{i=1}^{n} \sigma_{i} t r\left(\widetilde{W}_{i}^{T} \Gamma_{i}^{-1} \widehat{W}_{i}\right) .
\end{aligned}
$$

Then we have the following results:

$$
\begin{aligned}
\dot{V}_{\Xi} \leq & -\sum_{i=1}^{n} z_{i}^{T}(t)\left(K_{i}-\frac{1}{2} I^{m \times m}\right) z_{i}(t) \\
& -\sum_{i=1}^{n} \eta_{i} \widetilde{\chi}_{i}^{T}(t) \widetilde{\chi}_{i}(t)+\sum_{i=1}^{n} z_{i}^{T}(t) \widetilde{\chi}_{i}(t) \\
& -\sum_{i=1}^{n} z_{i}^{T}(t) \gamma_{i}(t)-\sum_{i=1}^{n} \widetilde{\chi}_{i}^{T}(t) \widetilde{W}_{i}^{T} S_{i} \\
& +\sum_{i=1}^{n} \widetilde{\chi}_{i}^{T}(t) \dot{\chi}_{i}(t)+\sum_{i=1}^{n} P_{i}\left(\bar{x}_{i}(t)\right) \\
& -\sum_{i=1}^{n} \sigma_{i} t r\left(\widetilde{W}_{i}^{T} \Gamma_{i}^{-1} \widehat{W}_{i}\right) .
\end{aligned}
$$

In addition, it is clear that there exists $\left\|S_{i}\right\| \leq \delta_{S i}, \delta_{S i}>0$; we have the following facts:

$$
\begin{aligned}
\sigma_{i} \operatorname{tr}\left(\widetilde{W}_{i}^{T} \Gamma_{i}^{-1} \widehat{W}_{i}\right) \leq & -\frac{\sigma_{i}}{2} \operatorname{tr}\left(\widetilde{W}_{i}^{T} \Gamma_{i}^{-1} \widetilde{W}_{i}\right) \\
& +\frac{\sigma_{i}}{2} \operatorname{tr}\left(W_{i}^{* T} \Gamma_{i}^{-1} W_{i}^{*}\right) \\
z_{i}^{T}(t) \widetilde{\chi}_{i}(t) \leq & \frac{\vartheta_{\xi i}}{2} z_{i}^{T}(t) z_{i}(t)+\frac{1}{2 \vartheta_{\xi i}}\left\|\widetilde{\chi}_{i}(t)\right\|^{2} \\
\widetilde{\chi}_{i}^{T}(t) \widetilde{W}_{i}^{T} S_{i} \leq & \frac{\delta_{S i} \varphi_{i}}{2}\left\|\widetilde{\chi}_{i}(t)\right\|^{2}+\frac{\delta_{S i}}{2 \varphi_{i}}\left\|\widetilde{W}_{i}\right\|^{2} \\
\widetilde{\chi}_{i}^{T}(t) \dot{\chi}_{i}(t) \leq & \frac{\pi_{i}}{2}\left\|\widetilde{\chi}_{i}(t)\right\|^{2}+\frac{1}{2 \pi_{i}}\left\|\dot{\chi}_{i}(t)\right\|^{2},
\end{aligned}
$$

where $\vartheta_{\xi i}>0$ and $\varphi_{i}>0$ are the constants, and then we have

$$
\begin{aligned}
\dot{V}_{\Xi} & \leq-\sum_{i=1}^{n}\left(\lambda_{\min }\left(K_{i}\right)-\frac{\vartheta_{\xi i}+1}{2}\right)\left\|z_{i}(t)\right\|-\sum_{i=1}^{n}\left(\eta_{i}\right. \\
& \left.-\frac{1}{2 \vartheta_{\xi i}}-\frac{\delta_{S i} \varphi_{i}}{2}-\frac{\pi_{i}}{2}\right)\left\|\widetilde{\chi}_{i}(t)\right\| \\
& -\sum_{i=1}^{n} \frac{\sigma_{i}}{2} \operatorname{tr}\left(\widetilde{W}_{i}^{T} \Gamma_{i}^{-1} \widetilde{W}_{i}\right)+\frac{1}{2} \\
& \cdot \sum_{i=1}^{n} \sum_{j=1}^{m}\left(1-16 \tanh ^{2}\left(\frac{z_{i j}(t)}{v_{i}}\right)\right) h_{i j}^{T}\left(\bar{x}_{i}(t)\right) \\
& \cdot h_{i j}\left(\bar{x}_{i}(t)\right)+\sum_{i=1}^{n} \frac{\sigma_{i}}{2} \operatorname{tr}\left(W_{i}^{* T} \Gamma_{i}^{-1} W_{i}^{*}\right) \\
& +\sum_{i=1}^{n} \frac{1}{2 \pi_{i}}\left\|\dot{\chi}_{i}(t)\right\| .
\end{aligned}
$$

Invoking Lemma 5, we obtain

$$
\dot{V}_{\Xi} \leq-\kappa V_{\Xi}+C
$$

$\kappa>0, C>0$ are defined in (50). Therefore, according to Lemma 4, we can conclude that the solution of the closedloop system remains within a compact subset.

\section{Application to Unmanned Helicopter System}

In this section, we will apply the proposed robust adaptive control strategy to solve the problem of attitude tracking for a class of uncertain unmanned helicopter systems.

The unmanned helicopters rigid-body dynamics consist of two parts, attitude angular dynamics and flapping dynamics. The unsteady aerodynamics bring out the time-delay nonlinear uncertainty; thus in this section we consider the attitude control for attitude control subject to time delay. Firstly, the attitude angular and angular velocity dynamics can be described as [26]

$$
\begin{aligned}
\dot{\Theta}(t)= & R(\Theta(t)) \Omega(t) \\
I \dot{\Omega}(t)= & -\Omega(t) \times(I \Omega(t))+\bar{A}(t) v_{c}(t) \\
& +h(\Theta(t), \Omega(t))+\bar{B}(t) \\
& +\Delta_{\Omega}(\Theta(t), \Omega(t))+d_{\Omega}(t),
\end{aligned}
$$

where $\Theta=[\psi, \theta, \phi]^{T} \in R^{3}$ indicates the attitude angle and $\Omega=[p, q, r]^{T} \in R^{3}$ represents the attitude angle velocity. $d_{\Omega}(t) \in R^{3}$ stands for the disturbance, and $\Delta_{\Omega} \in R^{3}$ stands for the uncertainties; $h(\Theta(t), \Omega(t)) \in R^{3}$ means the unknown 
nonlinear block of time delay. $v_{c}=\left[a, b, T_{T}\right]^{T} \in R^{3}, R(\Theta(t))$ denotes

$$
\begin{aligned}
& R(\Theta(t)) \\
& \quad=\left[\begin{array}{ccc}
1 & \sin \phi(t) \tan \theta(t) & \cos \phi(t) \tan \theta(t) \\
0 & \cos \phi(t) & -\sin \phi(t) \\
0 & \frac{\sin \phi(t)}{\cos \theta(t)} & \frac{\cos \phi(t)}{\cos \theta(t)}
\end{array}\right] .
\end{aligned}
$$

$\bar{A}$ and $\bar{B}$ denote

$$
\begin{aligned}
\bar{A} & =\left[\begin{array}{ccc}
-G_{R} & -z_{R} T_{R}+K_{\beta} & z_{T} \\
-z_{R} T_{R}+K_{\beta} & G_{R} & 0 \\
y_{R} T_{R} & x_{R} T_{R} & -x_{T}
\end{array}\right] \\
\bar{B} & =\left[\begin{array}{c}
-y_{R} T_{R} \\
x_{R} T_{R} \\
-G_{R}
\end{array}\right] \\
G_{R} & =C^{R}\left|T_{R}\right|^{1.5}+D^{R} .
\end{aligned}
$$

In the unmanned helicopter attitude control loop, the main rotor thrusts $T_{R}$ are fixed. $\left[x_{R}, y_{R}, z_{R}\right]^{T} \in R^{3}$ indicates the location of the main rotor relative to the center of gravity; $\left[x_{T}, y_{T}, z_{T}\right]^{T} \in R^{3}$ indicates the location of the tail rotor relative to the center of gravity. $K_{\beta} \in R$ means the stiffness of the rotor hub. $C^{R}$ and $D^{R}$ are constants, which are associated with the antitorque. The control design procedure can be presented as follows.

Step 1. Define the attitude angle tracking errors in the form of $\widetilde{z}_{\psi}(t)=\psi(t)-\psi_{r}(t), \widetilde{z}_{\theta}(t)=\theta(t)-\theta_{r}(t), \widetilde{z}_{\phi}(t)=\phi(t)-\phi_{r}(t)$, and $\widetilde{z}_{\Theta}(t)=\left[\widetilde{z}_{\psi}(t), \widetilde{z}_{\theta}(t), \widetilde{z}_{\phi}(t)\right]^{T}$, where $\psi_{r}(t), \theta_{r}(t)$, and $\phi_{r}(t)$ are the reference trajectories and $\Theta_{r}=\left[\psi_{r}(t), \theta_{r}(t), \phi_{r}(t)\right]^{T}$. Then the time derivative of $\widetilde{z}_{\Theta}(t)$ is achieved as

$$
\tilde{z}_{\Theta}=R(\Theta(t)) \Omega(t)-\dot{\Theta}_{r}(t) .
$$

According to the analysis in the previous section, the prescribed performance function is chosen as

$$
z_{\Theta j}(t)=\frac{1}{2} \ln \left[\frac{\left(\underline{\alpha}_{j}+\widetilde{z}_{\Theta j}(t) / \rho_{j}(t)\right)}{\left(\bar{\alpha}_{j}-\widetilde{z}_{\Theta j}(t) / \rho_{j}(t)\right)}\right], \begin{aligned}
& j=1, \ldots, 3, \\
& j=1,
\end{aligned}
$$

and the definition of $\rho_{j}(t)$ can be referred to in (6). Choose the virtual control signal as

$$
\begin{aligned}
& \Omega^{*}(t)=R^{-1}(\Theta(t)) \\
& \quad \cdot\left(-M_{\Theta}^{-1}(t) K_{\Theta} z_{\Theta}(t)+\dot{\Theta}_{r}(t)+M_{\Theta}^{-1}(t) N_{\Theta}(t)\right) .
\end{aligned}
$$

Choose the Lyapunov function:

$$
V_{\Theta}=\frac{z_{\Theta}^{T}(t) z_{\Theta}(t)}{2} .
$$

Step 2. Define the tracking errors $z_{p}(t)=p(t)-p_{r}^{*}(t)$, $z_{q}(t)=q(t)-q_{r}^{*}(t), z_{r}(t)=r(t)-r_{r}^{*}(t)$, and $\Omega^{*}(t)=$ $\left[p_{r}^{*}(t), q_{r}^{*}(t), r_{r}^{*}(t)\right]^{T}$. Using the neural network to compensate the uncertainties and time-delay terms, such that $\Delta_{\Omega}(\Theta(t), \Omega(t))+\gamma_{\Omega}(t)=\eta_{\Omega}^{-1} W_{\Omega}^{* T} S_{\Omega}+\varepsilon_{\Omega}^{*}$, assume that $\varepsilon_{\Omega}^{*}$ is the optimal approximate error, and define $\chi_{\Omega}(t)=\varepsilon_{\Omega}^{*}+d_{\Omega}(t)$. Design the neural updated law as

$$
\begin{aligned}
& \dot{\widehat{W}}_{\Omega}(t) \\
& \quad=\Gamma_{\Omega}\left(z_{\Omega}(t) S_{\Omega}(\Theta(t), \Omega(t))-\sigma_{\Omega} \Gamma_{\Omega}^{-1} \widehat{W}_{\Omega}(t)\right) .
\end{aligned}
$$

The DOB can be constructed in the form of

$$
\begin{aligned}
& \widehat{\chi}_{\Omega}(t)=w_{\Omega}(t)+\eta_{\Omega} \Omega(t) \\
& \dot{w}_{\Omega}(t)=-\eta_{\Omega} w_{\Omega}(t)-\eta_{\Omega}(-\Omega(t) \times(I \Omega(t)) \\
& \left.\quad+\bar{A}(t) v_{c}(t)+\bar{B}(t)+\eta_{\Omega}^{-1} \widehat{W}_{\Omega}^{T} S_{\Omega}+\eta_{\Omega} \Omega(t)\right) \\
& \quad+z_{\Omega}(t) .
\end{aligned}
$$

Based on the above design, the adaptive control law is achieved as

$$
\begin{gathered}
v_{c}(t)=\bar{A}^{-1}(t)\left(-K_{\Omega} z_{\Omega}(t)-R^{T}(\Theta) z_{\Theta}(t)+\dot{\Omega}^{*}(t)\right. \\
\left.+\Omega(t) \times(I \Omega(t))-\bar{B}(t)-\eta_{\Omega}^{-1} \widehat{W}_{\Omega}^{T} S_{\Omega}-\widehat{\chi}_{\Omega}(t)\right) .
\end{gathered}
$$

Choose the Lyapunov function:

$$
\begin{aligned}
V_{\Omega}= & \frac{1}{2} \Omega^{T}(t) \Omega(t)+\int_{t-\tau}^{t} P\left(\Theta\left(t_{\tau}\right), \Omega\left(t_{\tau}\right)\right) d t_{\tau} \\
& +\operatorname{tr}\left(\widetilde{W}_{\Omega}^{T} \Gamma_{\Omega}^{-1} \widetilde{W}_{\Omega}\right)+\frac{1}{2} \widetilde{\chi}_{\Omega}^{T}(t) \widetilde{\chi}_{\Omega}(t) .
\end{aligned}
$$

Similar to Theorem 7, we can conclude the following theorem.

Theorem 8. For the unmanned helicopter system (59), combining the DOB (67) and neural network update law (66), the control procedure can be achieved as (64) and (68); thus the closed-loop signals are bounded, and the output trajectories are satisfying the prescribed performance conditions.

For Theorem 8, the detailed proof can be referred to in Theorem 7 in the previous section.

\section{Simulation Results}

In this section, the numerical simulation about unmanned helicopter system is utilized for illustrating the effectiveness of the previous control method in this paper. Consider the unmanned helicopter system with the following parameters (Table 1) [24, 26].

Now let us look at the design of adaptive controller design, the $\mathrm{DOB}$ design parameters are chosen as $\eta_{\Omega}=6$. The neural update law has been given by 70 , and its parameters are chosen as $\Gamma_{\Omega}=0.1 \cdot \operatorname{diag}\{1,1,1,1,1,1,1\}, \sigma_{\Omega}=10, \mu=$ $[-3,-2,-1,0,1,2,3]$, and $c_{\Omega}=[1,1,1,1,1,2,3]$, and the controller design parameters are chosen as $K_{\Theta}=\{5,5,5\}$ and 
TABLE 1: Helicopter parameters.

\begin{tabular}{lcc}
\hline Symbol & Unit & Number \\
\hline$I$ & $\mathrm{~kg} \cdot \mathrm{m}^{2}$ & $\operatorname{diag}(0.18,0.34,0.28)$ \\
$m$ & $\mathrm{~kg}$ & 8.2 \\
$g$ & $\mathrm{~m} / \mathrm{sec}^{2}$ & 9.81 \\
$x_{T}$ & $\mathrm{~m}$ & -0.91 \\
$z_{T}$ & $\mathrm{~m}$ & -0.08 \\
$z_{R}$ & $\mathrm{~m}$ & -0.235 \\
$x_{R}$ & $\mathrm{~m}$ & 0 \\
$y_{R}$ & $\mathrm{~m}$ & 0 \\
$z_{R}$ & $\mathrm{~m}$ & 0 \\
$K_{\beta}$ & $\mathrm{N} \cdot \mathrm{m} / \mathrm{rad}$ & 52 \\
$C^{R}$ & $\mathrm{~m} / \sqrt{\mathrm{N}}$ & 0.004452 \\
$D^{R}$ & $\mathrm{~N} \cdot \mathrm{m}$ & 0.6304 \\
\hline
\end{tabular}

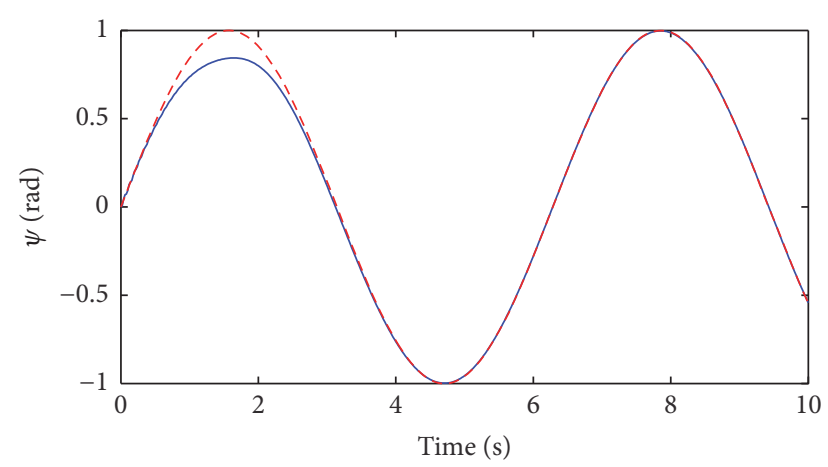

$-\psi$

FIGURE 1: Yaw angle.

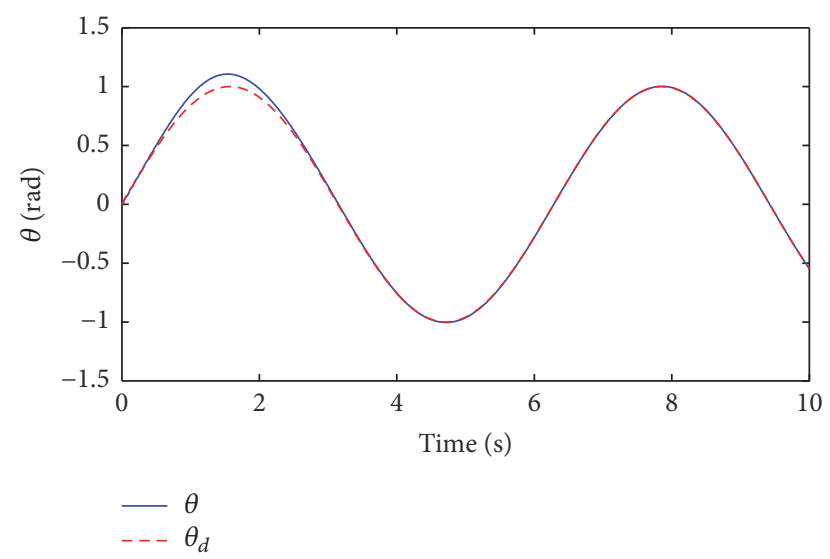

FIGURE 2: Pitch angle.

$K_{\Omega}=\{10,10,10\}$. The simulation environment is built under Matlab, and the simulation step is chosen as $\tau=0.02$. The initial conditions are chosen as follows: $\psi(0)=1 \mathrm{rad}, \psi(0)=$ $0 \mathrm{rad}$, and $\psi(0)=0 \mathrm{rad}$. The time-delay block is chosen in the form of $h(\Theta(t), \Omega(t))=\Omega(t-\tau)^{2}+\sin (0.1 \cdot \Omega(t-3 \tau))$. The external disturbance is chosen as $d_{\Omega}(t)=2 \cdot \sin (2 \cdot t+0.6)$.

From the curves in Figures $1-3$, it can be seen that the tracking trajectories soon reach the target trajectories, and

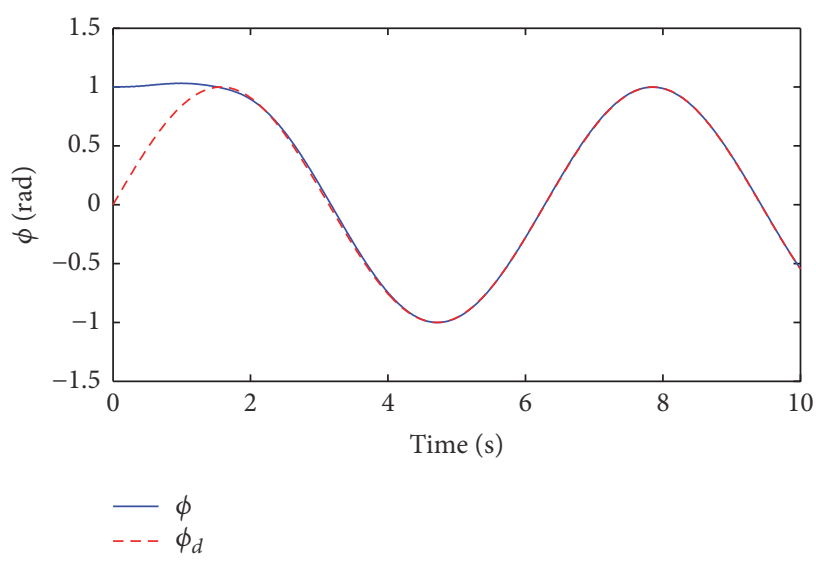

FIgURE 3: Roll angle.

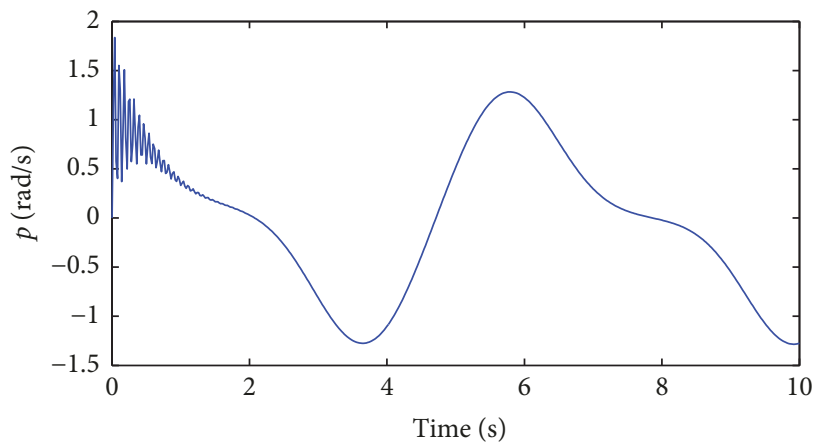

Figure 4: Yaw angular velocity.

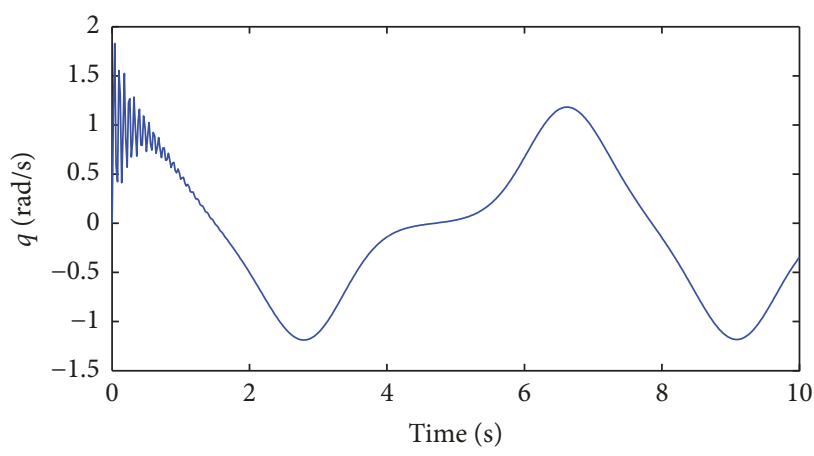

FIGURE 5: Pitch angular velocity.

the tracking errors are bounded in the appointed region. Figures 4-6 show the attitude angular velocities. Figures 7-9 show the control input signals. From the numerical simulation, we can conclude that the proposed control approach is valid for a class of uncertain unmanned helicopter systems with unsteady aerodynamics. Furthermore, it can be illustrated that the closed-loop system output signals are asymptotically tracking the ideal trajectories, and they are restricted in the region of output constraints.

\section{Conclusion}

In this paper, an adaptive prescribed performance control procedure has been proposed for a class of nonlinear timedelay systems with uncertainties, external disturbances, and 


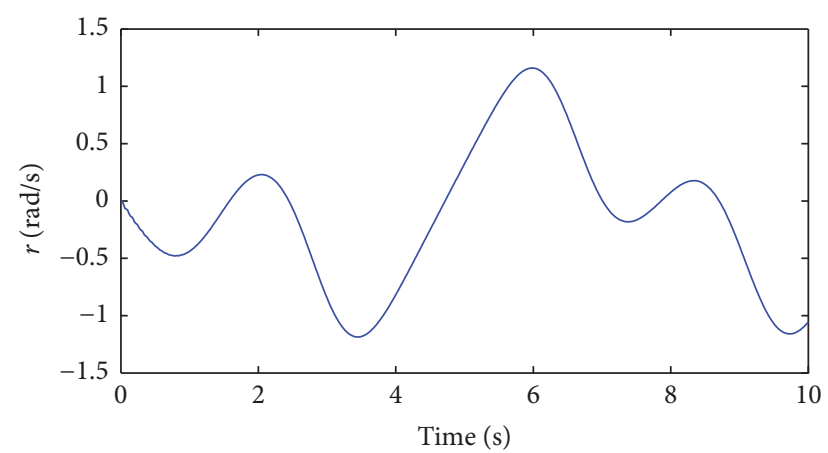

FIGURE 6: Roll angular velocity.

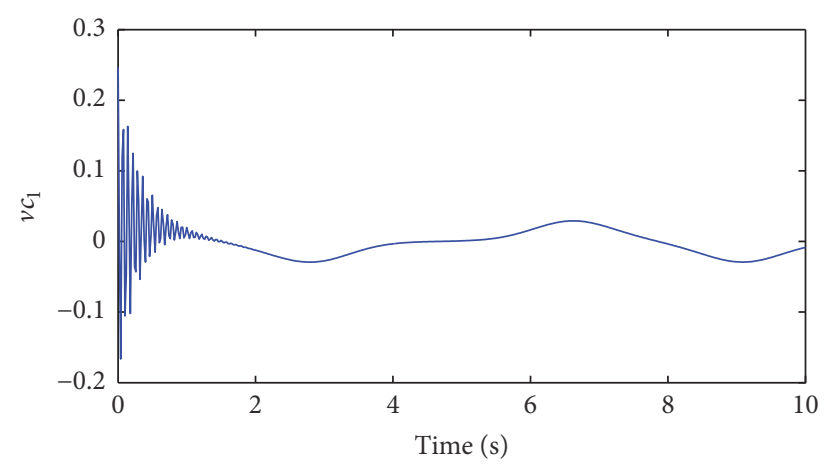

FIGURE 7: Control input signal $v_{c 1}(t)$.

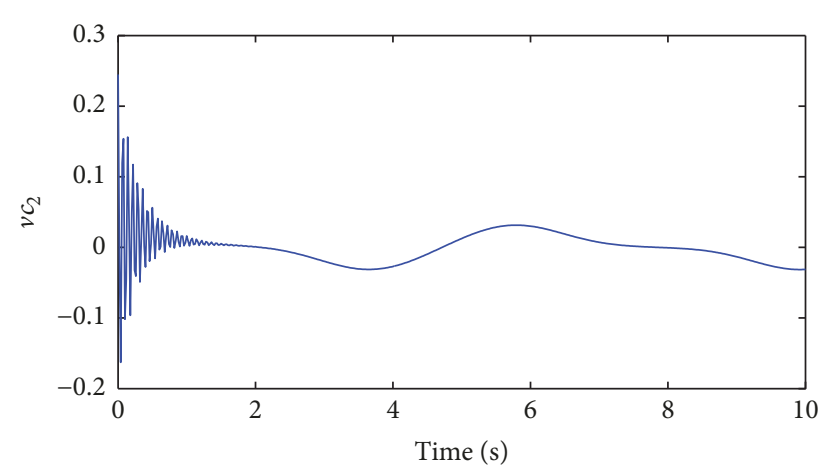

Figure 8: Control input signal $v_{c 2}(t)$.

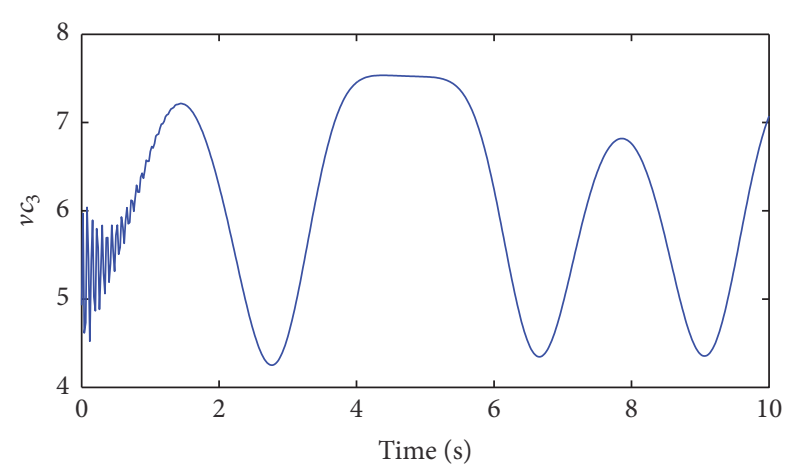

Figure 9: Control input signal $v_{c 3}(t)$. output constraints. Additionally, the robust controller has been applied to unmanned helicopter systems with unsteady aerodynamics. At last, the simulation illustrates that the proposed control approach is valid for the uncertain constrained time-delay system.

\section{Conflicts of Interest}

The authors declare that there are no conflicts of interest regarding the publication of this paper.

\section{Acknowledgments}

This article was supported by the National Natural Science Foundation of China (nos. 61573184, 61374212), the Fundamental Research Funds for the Central Universities (no. NE2016101), and the Aeronautical Science Foundation of China (no. 20165752049).

\section{References}

[1] P. Shi, E.-K. Boukas, and R. K. Agarwal, "Control of Markovian jump discrete-time systems with norm bounded uncertainty and unknown delay," Institute of Electrical and Electronics Engineers Transactions on Automatic Control, vol. 44, no. 11, pp. 2139-2144, 1999.

[2] J. Zhou, C. Wen, and W. Wang, "Adaptive backstepping control of uncertain systems with unknown input time-delay," Automatica, vol. 45, no. 6, pp. 1415-1422, 2009.

[3] S. S. Ge, F. Hong, and T. H. Lee, "Adaptive neural network control of nonlinear systems with unknown time delays," Institute of Electrical and Electronics Engineers Transactions on Automatic Control, vol. 48, no. 11, pp. 2004-2010, 2003.

[4] S. Tong, Y. Li, G. Feng, and T. Li, "Observer-based adaptive fuzzy backstepping dynamic surface control for a class of nonlinear systems with unknown time delays," IET Control Theory \& Applications, vol. 5, no. 12, pp. 1426-1438, 2011.

[5] Q. Zhou, P. Shi, S. Xu, and H. Li, "Observer-based adaptive neural network control for nonlinear stochastic systems with time delay," IEEE Transactions on Neural Networks and Learning Systems, vol. 24, no. 1, pp. 71-80, 2013.

[6] R. Yang and Y. Wang, "Finite-time stability analysis and H control for a class of nonlinear time-delay Hamiltonian systems," Automatica, vol. 49, no. 2, pp. 390-401, 2013.

[7] R. Yang and Y. Wang, "Finite-time stability and stabilization of a class of nonlinear time-delay systems," SIAM Journal on Control and Optimization, vol. 50, no. 5, pp. 3113-3131, 2012.

[8] M. Chen and G. Tao, "Adaptive Fault-Tolerant Control of Uncertain Nonlinear Large-Scale Systems With Unknown Dead Zone," IEEE Transactions on Cybernetics, vol. 46, no. 8, pp. 1851$1862,2016$.

[9] M. Chen, S. S. Ge, and B. V. E. How, "Robust adaptive neural network control for a class of uncertain MIMO nonlinear systems with input nonlinearities," IEEE Transactions on Neural Networks and Learning Systems, vol. 21, no. 5, pp. 796-812, 2010.

[10] M. Chen, P. Shi, and C. C. Lim, "Adaptive neural fault-tolerant control of a 3-dof model helicopter system," IEEE Transactions on Systems, Man, and Cybernetics: Systems, vol. 46, no. 2, pp. 260-270, 2015. 
[11] M. Chen, P. Shi, and C.-C. Lim, "Robust constrained control for MIMO nonlinear systems based on disturbance observer," Institute of Electrical and Electronics Engineers Transactions on Automatic Control, vol. 60, no. 12, pp. 3281-3286, 2015.

[12] J. Yang, W.-H. Chen, S. Li, L. Guo, and Y. Yan, "Disturbance/Uncertainty Estimation and Attenuation Techniques in PMSM Drives - A Survey," IEEE Transactions on Industrial Electronics, vol. 64, no. 4, pp. 3273-3285, 2017.

[13] J. Zhang, X. Liu, Y. Xia, Z. Zuo, and Y. Wang, "Disturbance Observer-Based Integral Sliding-Mode Control for Systems with Mismatched Disturbances," IEEE Transactions on Industrial Electronics, vol. 63, no. 11, pp. 7040-7048, 2016.

[14] B. Xu, "Disturbance observer-based dynamic surface control of transport aircraft with continuous heavy cargo airdrop," IEEE Transactions on Systems, Man, and Cybernetics: Systems, 2016.

[15] M. Chen, "Constrained control allocation for overactuated aircraft using a neurodynamic model," IEEE Transactions on Systems, Man, and Cybernetics: Systems, vol. 46, no. 12, pp. 16301641, 2016.

[16] R. Li, M. Chen, and Q. Wu, "Adaptive neural tracking control for uncertain nonlinear systems with input and output constraints using disturbance observer," Neurocomputing, vol. 235, pp. 2737, 2017.

[17] B. Niu and J. Zhao, "Tracking control for output-constrained nonlinear switched systems with a barrier Lyapunov function," International Journal of Systems Science, vol. 44, no. 5, pp. 978985, 2013.

[18] Y.-J. Liu and S. Tong, "Barrier Lyapunov Functions-based adaptive control for a class of nonlinear pure-feedback systems with full state constraints," Automatica, vol. 64, pp. 70-75, 2016.

[19] W. He, Z. Yin, and C. Sun, "Adaptive Neural Network Control of a Marine Vessel With Constraints Using the Asymmetric Barrier Lyapunov Function," IEEE Transactions on Cybernetics, 2016.

[20] C. P. Bechlioulis and G. A. Rovithakis, "Robust adaptive control of feedback linearizable MIMO nonlinear systems with prescribed performance," Institute of Electrical and Electronics Engineers Transactions on Automatic Control, vol. 53, no. 9, pp. 2090-2099, 2008.

[21] S. Tong, S. Sui, and Y. Li, "Fuzzy adaptive output feedback control of MIMO nonlinear systems with partial tracking errors constrained," IEEE Transactions on Fuzzy Systems, 2014.

[22] K. Peng, G. Cai, B. M. Chen, M. Dong, K. Y. Lum, and T. H. Lee, "Design and implementation of an autonomous flight control law for a UAV helicopter," Automatica, vol. 45, no. 10, pp. 2333 2338, 2009.

[23] G. W. Cai, B. M. Chen, X. Dong, and T. H. Lee, "Design and implementation of a robust and nonlinear flight control system for an unmanned helicopter," Mechatronics, vol. 21, no. 5, pp. 803-820, 2011.

[24] J. Fu, W.-H. Chen, and Q.-X. Wu, "Chattering-free sliding mode control with unidirectional auxiliary surfaces for miniature helicopters," International Journal of Intelligent Computing and Cybernetics, vol. 5, no. 3, pp. 421-438, 2012.

[25] C. Liu, W.-H. Chen, and J. Andrews, "Model predictive control for autonomous helicopters with computational delay," in Proceedings of the UKACC International Conference on CONTROL 2010, pp. 656-661, UK, September 2010.

[26] R. Li, M. Chen, Q. Wu, and J. Liu, "Robust adaptive tracking control for unmanned helicopter with constraints," International Journal of Advanced Robotic Systems, vol. 14, no. 3, 2017. 


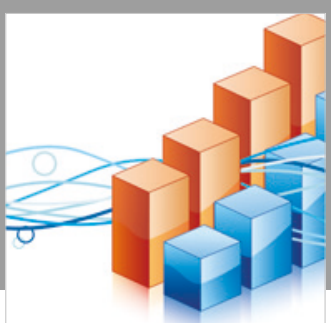

Advances in

Operations Research

\section{-n-m}
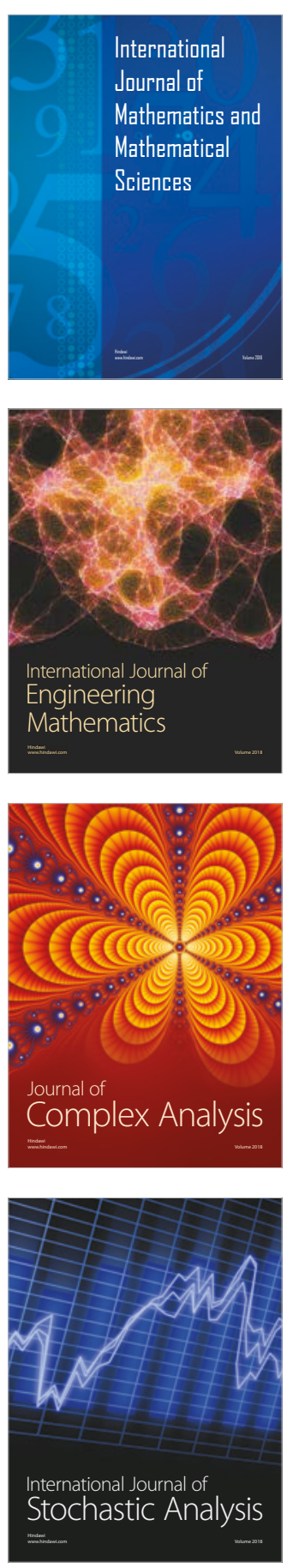
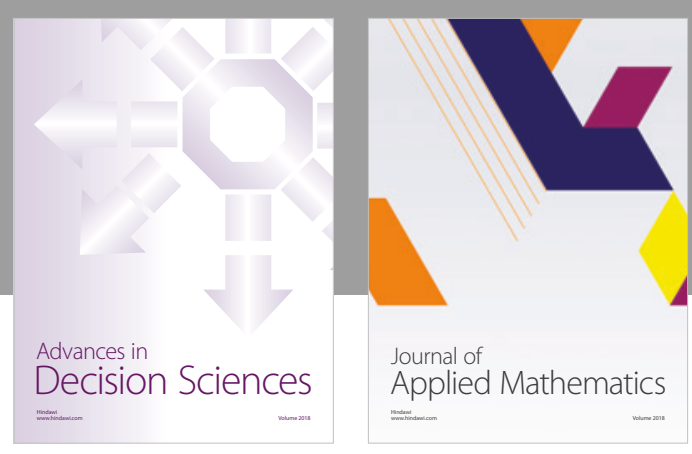

Journal of

Applied Mathematics
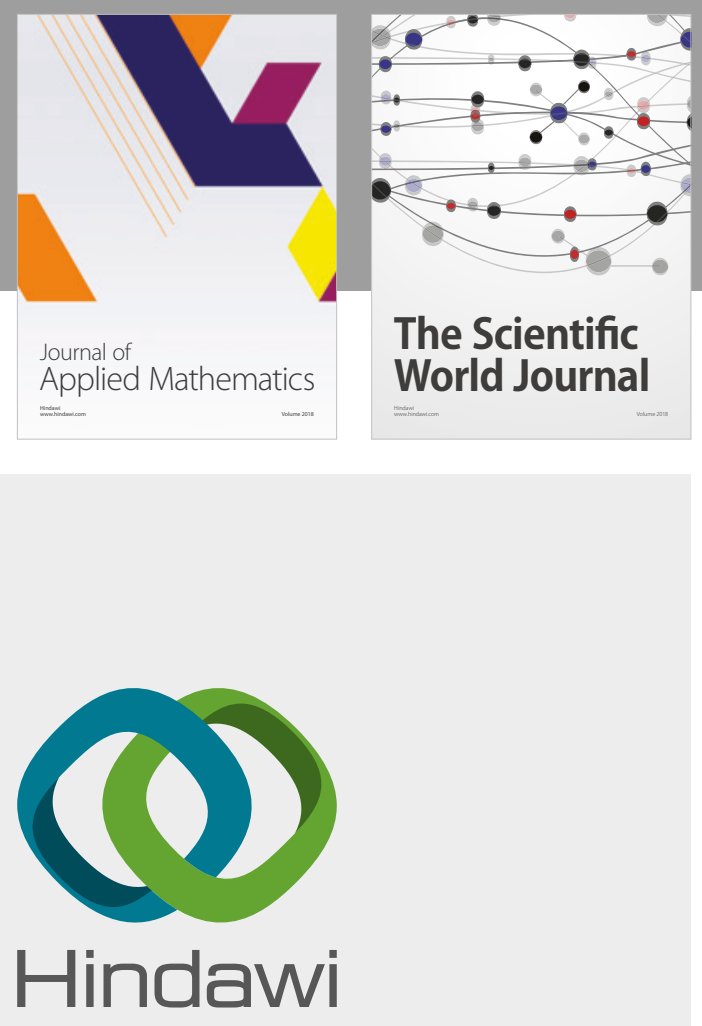

Submit your manuscripts at

www.hindawi.com

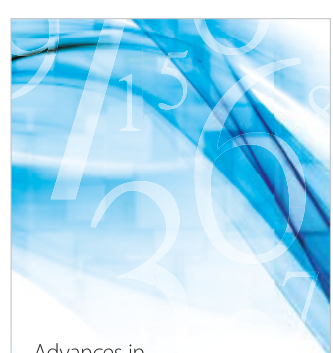

Advances in
Numerical Analysis
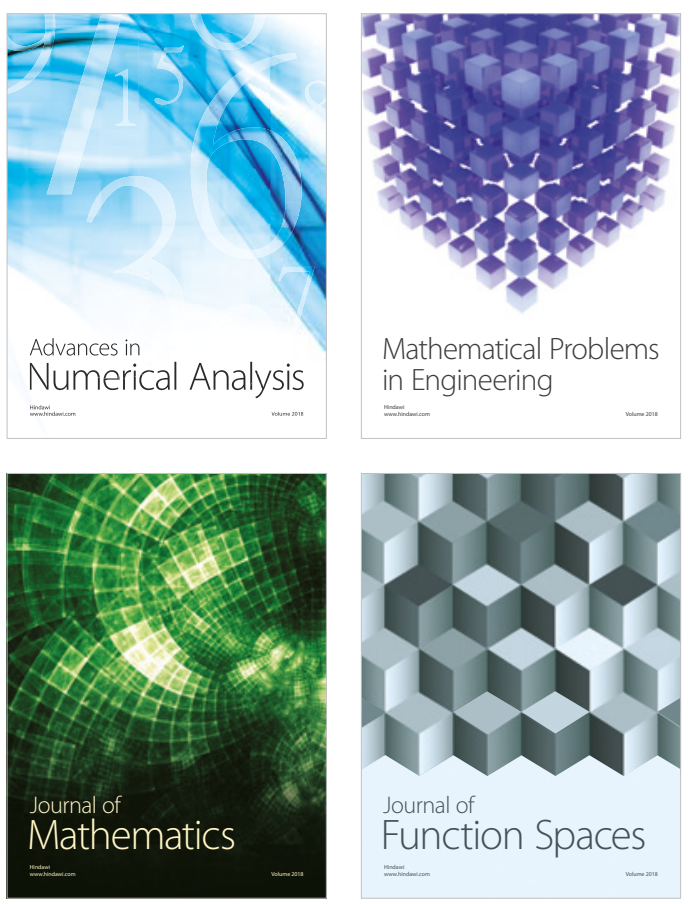

Mathematical Problems in Engineering

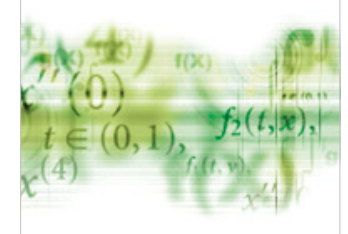

International Journal of

Differential Equations

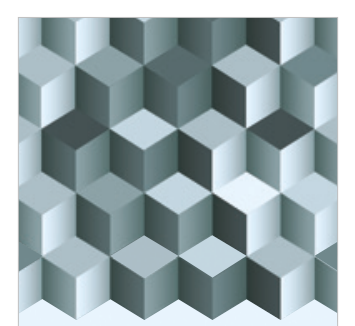

Journal of

Function Spaces

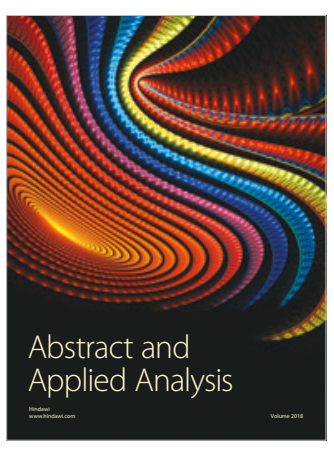

The Scientific

World Journal

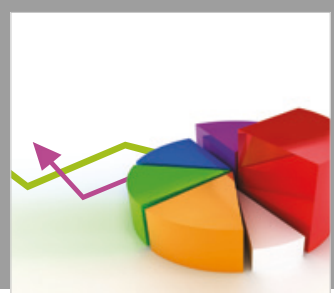

Journal of

Probability and Statistics
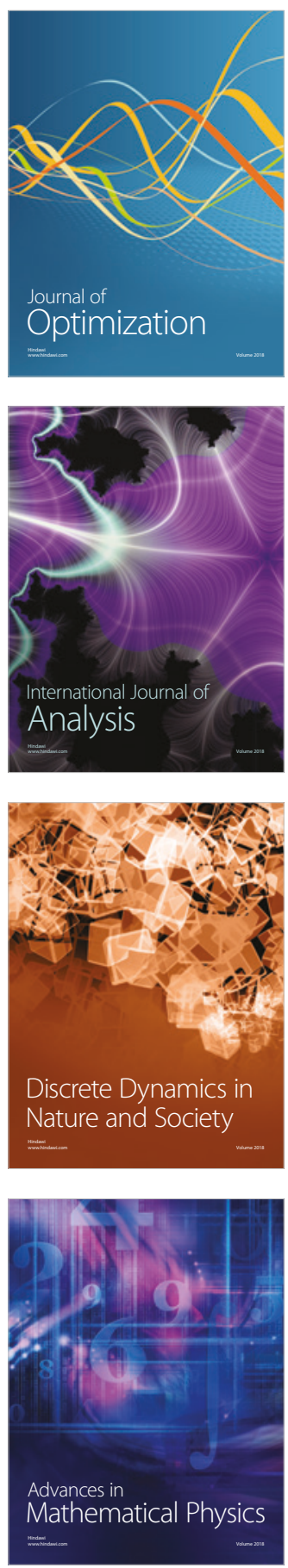